\title{
Magnetic Fields and Scintillator Performance
}

\author{
Dan Green and Anatoly Ronzhin \\ Fermi National Accelerator Laboratory \\ P.O. Box 500, Batavia, Illinois 60510 \\ Vasken Hagopian \\ Florida State University \\ Tallahasse, Florida 32306
}

June 1995 


\section{Disclaimer}

This report was prepared as an account of work sponsored by an agency of the United States Government. Neither the United States Government nor any agency thereof, nor any of their employees, makes any warranty, express or implied, or assumes any legal liability or responsibility for the accuracy, completeness, or usefulness of any information, apparatus, product, or process disclosed, or represents that its use would not infringe privately owned rights. Reference herein to any specific commercial product, process, or service by trade name, trademark, manufacturer, or otherwise, does not necessarily constitute or imply its endorsement, recommendation, or favoring by the United States Government or any agency thereof. The views and opinions of authors expressed herein do not necessarily state or reflect those of the United States Government or any agency thereof. 
FERMILAB TM-1937, July 1995

\author{
MAGNETIC FIELDS AND \\ SCINTILLATOR PERFORMANCE \\ Dan Green, Anatoly Ronzhin \\ Fermilab \\ Vasken Hagopian \\ Florida State University
}

\title{
INTRODUCTION
}

Experimental data have shown that the light output of a scintillator depends on the magnitude of the externally applied magnetic fields $(1,2,3,4)$, and that this variation can affect the calorimeter calibration and possibly resolution (5). The goal of the measurements presented here is to study the light yield of scintillators in high magnetic fields in conditions that are similar to those anticipated for the LHC CMS detector $(6,7)$. Two independent measurements were performed, the first at Fermilab and the second at the National High Magnetic Field Laboratory at Florida State University.

\section{FERMILAB MEASUREMENTS}

The tests were performed in the FNAL proton pool area magnet facility. The facility has a conventional magnet with a maximum field magnitude of 24300 gauss. The non uniformity of the magnetic field within the tested scintillators samples was less than 3\%. The samples of scintillator to be tested were installed between the poles of the magnet (fig.1). The scintillators were either directly coupled with optical clear fiber or coupled through wave length shifting (WIS) fiber in optical contact with the scintillators through air. Each scintillator was viewed from opposite sides by 2 clear fibers (fig.1). The fibers were connected to photomultipliers (PMT,s, XP2013B type). 
Two types of scintillators based on polystyrene were tested: Kuraray SCSN38 and a scintillator prepared in Protvino, IHEP by the injection molding method. Y11 (when used as WLS fiber) was fused to clear fiber inside of small glass tubes by a heating method.

The size of each scintillator was $31 \times 21 \times 4 \mathrm{~mm}^{3}$. To check the behavior of the WLS fiber in a magnetic field, scintillator of size $31 \times 250 \times 4 \mathrm{~mm}^{3}$ was used. The WLS fiber was optically connected to the $31 \times 4 \mathrm{~mm}^{2}$ side of the scintillators in all cases. The diameter of the clear fiber and the WLS fiber was $1 \mathrm{~mm}$. The lengths of the clear fibers were 2 meters. The length of $Y 11$ fiber was $7 \mathrm{~cm}$.

The scintillators were wrapped (together with fibers) first with white paper then light protected with black paper. The clear fibers were produced in light protected cladding. Clear fibers were glued to the scintillator (inside small holes in the opposite corners) when scintillators were tested without WLS fiber. The scintillators were irradiated by a Ra226 source placed on the flat surface of the scintillator inside of the magnetic field volume as shown in fig.1.

The single electron method was used to measure the light yield of the scintillators as in reference (3). The setup is shown in fig.2. In our geometry the initial amount of light is several photoelectrons. Nevertheless, to get to a single electron mode of measurement some additional suppression of light was applied by changing and fixing the distance between clear fiber and the photocathode of the photomultipliers.

The measured values are the single counting rate ( $\mathrm{Nl}$ and $\mathrm{Nr}$ ) for each PMT and the coincidence of both of them (Nlr). The ratios $\mathrm{Nlr} / \mathrm{Nl}$ and $\mathrm{Nlr} / \mathrm{Nr}$ are used to measure the number of photoelectrons according to the formula:

$$
\mathrm{N} \text { pe }=-0.5[\ln (1-\mathrm{Nlr} / \mathrm{Nl})+\ln (1-\mathrm{Nlr} / \mathrm{Nr})] \text {. }
$$

The relative light yield change for the current value of the magnetic field B was calculated from the expression:

$$
\text { delta } L / L=[\text { Npe }(B)-N p e(B=0)] / \text { Npe }(B=0)
$$

The maximum value of the efficiency $(\mathrm{Nlr} / \mathrm{Nl}, \mathrm{Nlr} / \mathrm{Nr})$ was adjusted to be less a than $7 \%$ in the measurements.

Some data were obtained using a PMT by measuring the direct current (DC) for comparison. In that case, the scintillator was irradiated by a Cs 137 radioactive source $(10 \mathrm{mC})$ and the current was measured by a microammeter. 
The magnitude of the magnetic field was measured by a Hall probe. The value of the magnetic field around the PMT was less than 1 gs. PMT's were well shielded by using a magnetic shield. Nevertheless, the direct influence of the magnetic field (up to 24300 gauss) on the PMT was tested and no influence was observed.

SCSN38 connected to clear fiber was tested first in the magnetic field (fig.3). The same was done when SCSN38 was optically connected to clear fibers through Y11 WLS-fiber. The molded scintillator (IHEP, Protvino) optically connected with Y11 fiber was also tested. The SCSN38 sample of size $31 \times 250 \times 4 \mathrm{~mm}^{3}$ and Y11 fiber was installed in the magnet. The Y11 was inside of the field and the Ra226 source was outside. The source was well shielded (less than 3 gs). The goal was to see some difference when Y11 fiber coupled with scintillator was adjusted inside the magnetic field and radioactive source was outside. The data are shown in fig. 3.

\section{BACKGROUND}

The main backgrounds for this setup were PMT noise and accidental coincidences. The cosmic counting rate Nlr was less than $0.1 \%$ compared to the presence of the Ra226 source. The PMT noise was not affected by the magnetic field. The value of the noise was around $7 \%$ and $3 \%$ for the left and right PMT respectively. These values were measured without the Ra226 source. Noise was subtracted from the data using the measurements with and without the Ra226 when the magnetic field was applied.

Long term changes of the noise counting rate were observed especially when starting the measurement immediately after high voltage was placed on the PMT. Therefore, all measurement were made only after 3 hours of operation with the PMT,s under HV. No more than a $10 \%$ change of the noise counting rate was observed during this stage of measurement.

To get the single electron counting rate dependence on magnetic field under Ra226 irradiation for each PMT the noise was subtracted from the data. The value of the accidental coincidence rate was measured simultaneously with data collection by using an additional coincidence circuit and delay line (16 meters of $50 \mathrm{Ohm}$ cable). The duration of the shaped output signals for each PMT was $30 \mathrm{nsec}$. The fraction of accidental coincidence was around $2 \%$ with respect to the Nlr counting rate. 
The total error of all measured sources of background for collected data was estimated to be $0.7 \%$ including long term non stability. The data collected for different runs were reproducible at the same level. The statistical error was less than this value. Some test data obtained by the DC method were consistent with those reported here at the level of $1 \%$. The accuracy of the direct current measurements was mainly related to the stability of the PMT's.

\section{FLORIDA STATE UNIVERSITY MEASUREMENTS}

Two magnets were used for these measurements. A warm coil small magnet with a variable opening capable of a maximum of $8 \mathrm{Kg}$ and the second magnet at the National High Magnetic Field Laboratory at Florida State University with a $5 \mathrm{~cm}$ bore capable of a $200 \mathrm{Kg}$ field. For these measurements a Co60 source was used. The effect of the magnetic field on the Co60 source was tested with the source both inside and outside the small magnet and no effect was observed. Finally the low field conventional magnet was employed to measure the light yield increase using relativistic cosmic muons. In addition a short wavelength UV light was used to excite the fluors in the scintillators and fiber to determine the source of the light yield increase.

\section{DATA}

The magnet at the National High Magnetic Field Laboratory has a 51 $\mathrm{mm}$ bore and is capable of a $200 \mathrm{Kg}$ field. For this test five types of scintillators and two types of WLS fibers were used: SCSN38 and SCSN81 made by Kuraray, BC404A and BC499-52 made by BICRON, NE110 made by Nuclear Enterprises and the WLS fibers BCF91A made by BICRON and Y11 by Kuraray (ref.8). Both BCF91A and Y11 use the same fluor K27. In addition, the same type scintillators and fibers were also measured in a small magnet with a maximum field strength of $8 \mathrm{Kg}$. In the small magnet measurements, the Co60 source was placed both outside and inside the magnetic field and the results were the same. The conclusion is that in up to $8 \mathrm{Kg}$ field the activity of the source does not change. It was impossible to perform the same test in the $200 \mathrm{Kg}$ magnet. The light yield increase in both magnets were the same between 1 and $8 \mathrm{Kg}$ within $\pm 0.5 \%$ error. The zero current field of the $200 \mathrm{Kg}$ magnet was unknown and probably about 10 gauss. 
In the small magnet, the scintillators were directly coupled to phototubes by means of UV transmitting light guides. The phototube was well shielded from magnetic fields by iron and mu metal cylinders. When the WLS fibers were tested, the fiber was connected directly to the phototube. In the $200 \mathrm{Kg}$ magnet, the scintillators were coupled to the WLS fibers and read with a green sensitive Hamamatsu phototube, R580-17. The length of the fibers were 4 meters with about 3.5 meters outside of the magnet. The scintillators were cut in a circle of $46 \mathrm{~mm}$ diameter and a $2 \mathrm{~mm}$ deep groove was machined near the edge, $1 \mathrm{~mm}$ inside the circumference. Each WLS fiber was 8 meters long with the middle of the fiber placed in the groove of the scintillator. Each scintillator edge was painted white with BICRON Corporation BC620 paint that resulted in an increase in light yield of about 50\%. The assembly contained an 8 micro curie Co60 source sandwiched between two scintillators. An Intel 486 based computer with an $\mathrm{ADC}$ card read the current of the phototube (typically about 300 namp) about 30,000 times. The scintillator and fiber were also excited by a short wavelength UV light source in both the 8 and $200 \mathrm{Kg}$ magnet.

The largest source of error in these type of measurements is the fringe field of the magnets at the phototubes and the temperature stability of the photocathode. For blue light the temperature stability is not very critical as the photocathode quantum efficiency is quite stable. For green wavelengths, such as the K27 fluor which peaks at $495 \mathrm{~nm}$, the quantum efficiency of about $18 \%$ is very temperature sensitive. The best procedure is to perform the measurements quickly on the time scale much less than temperature variations. Figure 4 shows the light yield increase due to magnetic fields of up to 140 Kgauss. Even though the measurements were performed up to 200 Kgauss the shielding of the phototubes was not perfect and a $2 \%$ drop in light yield was observed which was attributed to the fringe field of the magnet at the phototube. The point to point errors are usually less than $0.5 \%$, while the overall errors were less than $1 \%$.

Both SCSN38 and SCSN81 are polystyrene and show the same light yield increase. NE110 is made of polyvinyltoluene. The other two scintillators are mixtures. Repeated measurements gave very consistent results. In general terms, when excited by a Co60 radioactive source, the light yield increase occurs in the first $20 \mathrm{Kg}$ and then saturates above that value. The curves are to guide the eye 
and are not fits. Several thicknesses of scintillators were used and the light yield increase was independent of thickness. The magnetic field was ramped up to $200 \mathrm{Kg}$ and down to zero and the light yield results were the same to within our measurement errors of $1 \%$. The plots show the average of the ramp up and down results.

To measure the light yield increase due to the fluors of scintillators and fibers, the excitation was performed by short wavelength (about $300 \mathrm{~nm}$ ) UV light transported by a 4 meter long quartz fiber. The quartz fiber illuminated the blue scintillator, which was coupled to the green WLS fiber. Since UV lights are not uniform in time, another quartz fiber was connected to the source and the other end illuminated a second phototube, that was used for calibration. The lower portion of figure 4 shows that the light yield increase due to fluor excitation as a function of magnetic field is essentially consistent with zero $\pm 1 \%$ up to $140 \mathrm{Kg}$ of magnetic field. This fact, and the fact that the WLS displays no increase (see fig.3) indicates that the light increase occurs in the primary excitation of the scintillator.

Finally, as a check of the Co60 excitation, the small magnet aperture was opened to $11 \mathrm{~cm}$ and a flat $10 \mathrm{~cm}$ scintillator was placed in the magnetic field. The maximum field tested in this configuration was $1.5 \mathrm{Kg}$. The cosmic muons used for this measurement were required to pass through $5 \mathrm{~cm}$ of lead to ensure the presence of relativistic muons. Since it takes about 50 hours to collect enough cosmic muon data, during which time the sensitivity of the phototube can change, another scintillator outside the magnet was also connected with the same phototube but triggered with a second cosmic muon telescope. Both telescopes had 3 scintillators in coincidence in a vertical setup as follows from top to bottom: 1) telescope tile, 2) sample tile, 3) telescope tile, 4) $5 \mathrm{~cm}$ lead, 5) telescope tile. At $1.5 \mathrm{Kg}$ the light yield increase due to the cosmic muons was the same (2.6\% vs $2.7 \%)$ as that measured by Co60 excitation.

\section{DISCUSSION}

The whole set of world data show the following: 1. The polystyrene based scintillators irradiated by radioactive sources show a relative light yield increase up to $1-1.8 \%$ for a magnitude of the magnetic field up to $0.1 \mathrm{Kg}$. There follows a 
plateau up to $1 \mathrm{Kg}$. One can then observe further growth between 1 $\mathrm{Kg}$ and $20 \mathrm{Kg}$. The only data up to a $200 \mathrm{Kg}$ field show a second plateau after $20-30 \mathrm{Kg}$. This means that some additional mechanism causes the increase in the light yield of scintillator in the range 1 $30 \mathrm{Kg}$. The data doesn't depend on the magnetic field orientation. It is also not strongly dependent on the source of irradiation used (less than $1 \%$ difference for gammas or electrons).

2. For the magnitude of the magnetic field less than $2 \mathrm{Kg}$, the data of the DESY group show the same behavior for a sampling scintillator (SCSN38) calorimeter irradiated by 2 and $6 \mathrm{GeV}$ electrons and for a piece of the same scintillator irradiated by a Fe55 source. Our data obtained with a Ra226 source are in good agreement with the DESY data for magnetic fields up to $5 \mathrm{Kg}$. For larger magnitudes of the field our data are lower and are $5.3 \%$ for a field of $15 \mathrm{Kg}$. The maximum DESY field point of $14 \mathrm{Kg}$ has a $8.5 \%$ light yield shift. The data (9) obtained for Shashlik calorimeter also show a larger light increase (e.g. $9.2 \%$ at $24.5 \mathrm{Kg}$ ).

Our data indicate "a plateau" value around $6.3 \%$. One can note that our data are consistent with the high field data (10) in the range $0-24.3 \mathrm{Kg}$. It's difficult to make a quantitive conclusion from the data because the measurements were carried out under different conditions. Nevertheless, measurements with calorimeters in electron beams show several percent more light yield at the highest field in comparison with a single plate irradiated by a source (fig.5). Evidently both beams and source data are important for the calibration of a calorimeter. Therefore it will be crucial to get both data sets for the same calorimeter, as is planned for CMS hadron calorimeter.

For a hadron calorimeter it's also very important to measure the response to both hadrons and electrons in a magnetic field. Such measurements for CMS are planned to be done at CERN this summer in the $\mathrm{H} 2$ test beam.

3. It seems to be well established that there is no influence of magnetic field (for magnitude up to $140 \mathrm{Kg}$ ) when scintillator or WLS is irradiated by ultraviolet light. So the light yield increase is not due to the fluors. 


\section{ACKNOWLEDGMENTS}

We would like to thank Bill Foster, Jim Freeman, Mike Lamm and Adam Para for useful discussions. We also appreciate Virgil Barnes, Steve Gourlay and Julius Lentz for technical support. We would like to thank Prof. J. Crow Director and Dr. S. Hannahs liason physicist of the National High Magnetic Field Laboratory for the help in using the high field magnets. We would also like to acknowledge the Florida State University physicists Dr. J. H. Goldman, Dr. K. Johnson and Miss H. Witaker. 
BIBLIOGRAPHY

1.S.Bertolucci et al. NIM, A254, 1987, p.561-562.

2.J.P.Cumalat et al. NIM, A293, 1990, p.606-614.

3.D.Blomker et al. NIM, A311, 1992, p.505-511.

4.J.Manuisch et al. NIM, A312, 1992, p.451-456.

5.D.Green, preprint FNAL-TM-1823, 1993.

6.Compact Muon Solenoid (CMS) Detector, US Collaboration, Letter of Intent, April 22, 1994.

7.R\&D Proposal of a Hadron Calorimeter for High Magnetic Fields, CERN / DRDC / 94-22, DRDC / P 57, 9th May 1994.

8.BICRON Corporation, 12345 Kingsman Rd, Newbury, Oh. 44065, USA Kuraray Co. Ltd, Shuwa Higashi Yaesu Bldg, 10 Fl. 9-1, 2-Chome, Chuo-Ku, Tokyo, 104, Japan

NE Technology Ltd, Bankhead Medway, Sighthill Indust Est, Edinburgh, Britain

9.L.Dobrzynski et al. Shashlik Calorimetry. A combined Shashlik + Preshower detector for LHC. RD36, approved January 93, CERN. 10.V.Hagopian et al. Scintillators and wavelength shifting fibers in magnetic fields of up to 20 Tesla. To be published in the Proceedings of the V International Conference on Calorimetry in High Energy Physics (1994). 
FIGURES.

1.Front and side views of the setup.

Pole - poles of the magnet, scint - tested scintillator,

Ra226 - radioactive source, PM - photomultipliers.

2.Scheme of the measurements. Magn.field - external magnetic field, scint. - tested scintillator, Ra226 - radioactive source, PM - photomultipliers, SH - shapers, coinc - coincidence circuit, $\mathrm{Nl}, \mathrm{Nr}$ - left, right counting rates, Nlr coincidence counting rate.

3.Dependence of relative light yield of scintillators on the value of the magnetic field.

4. Relative light yield change of scintillators in magnetic fields with excitation by a) Co60 b) short wavelength UV.

5.The same as 3,4 for world data. 


\section{FRONT VIEW}

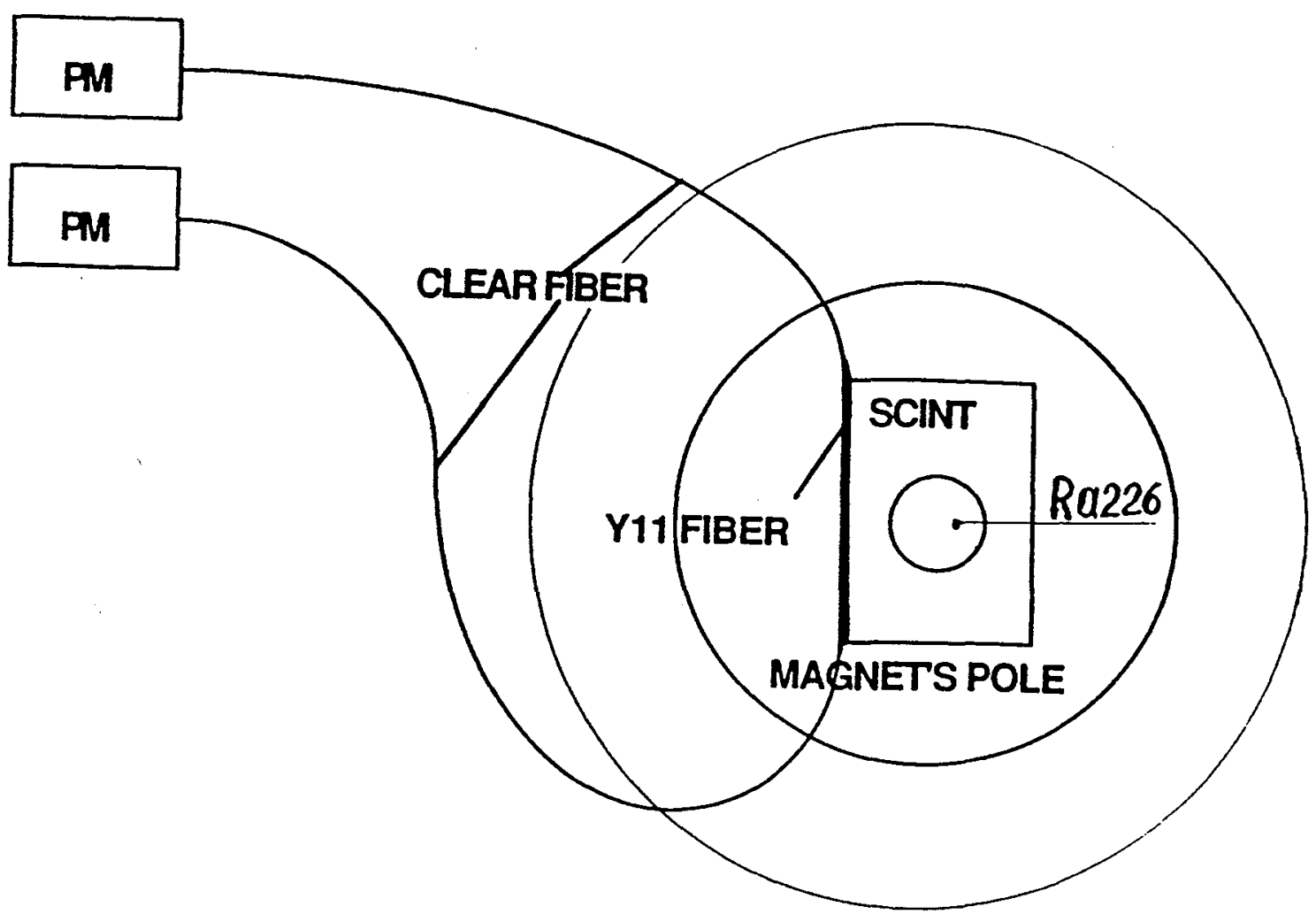

SIDE VIEW

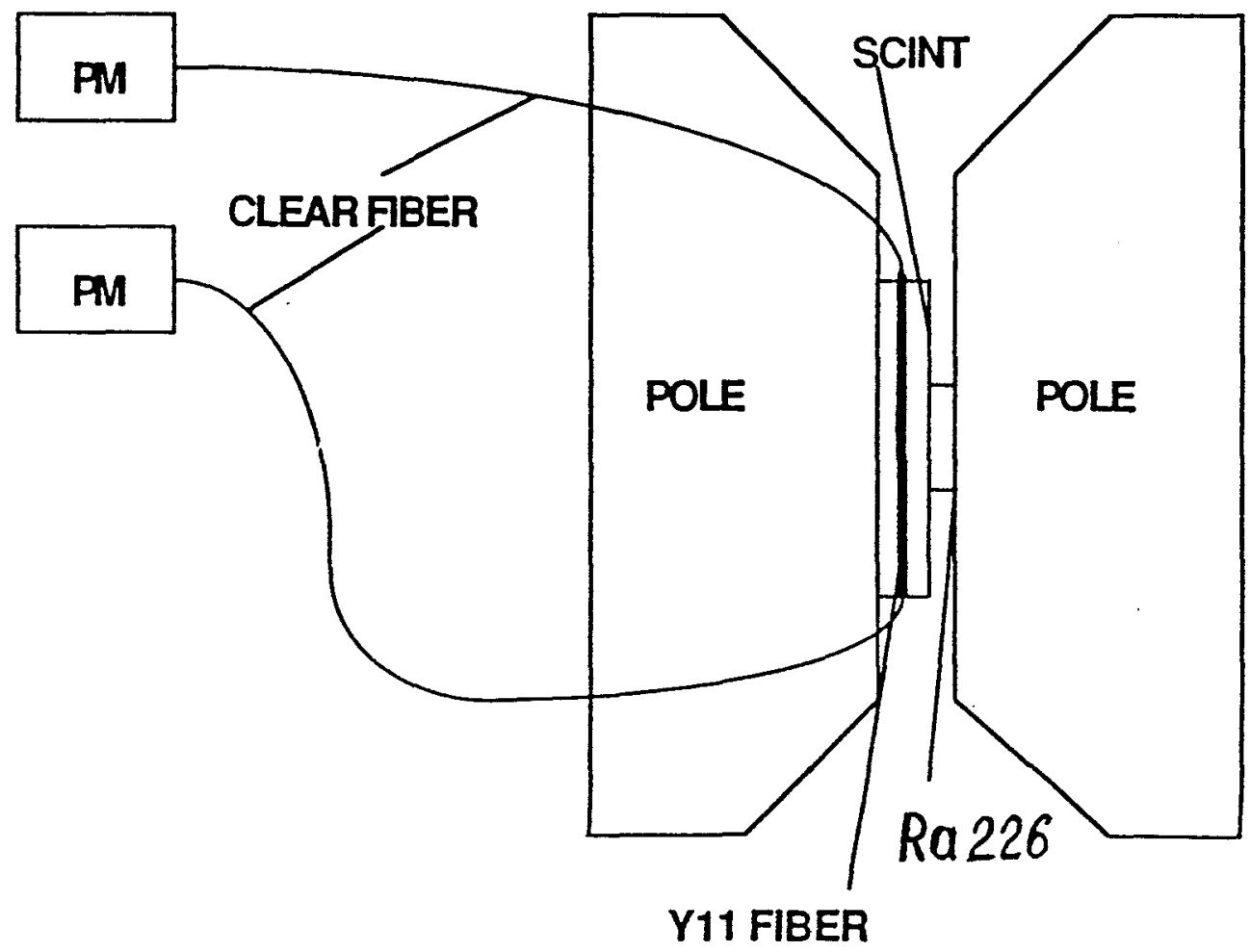

Fig.1. 


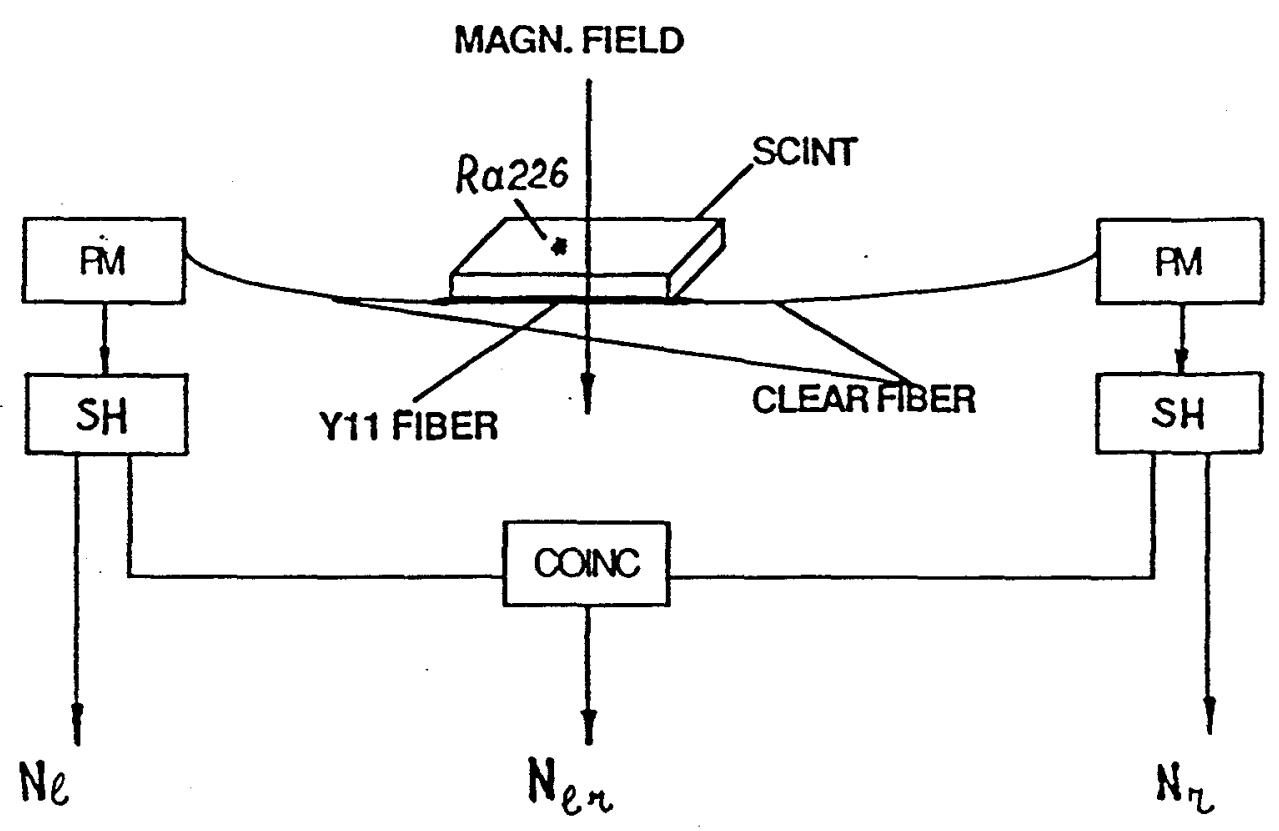

Fig. 2. 


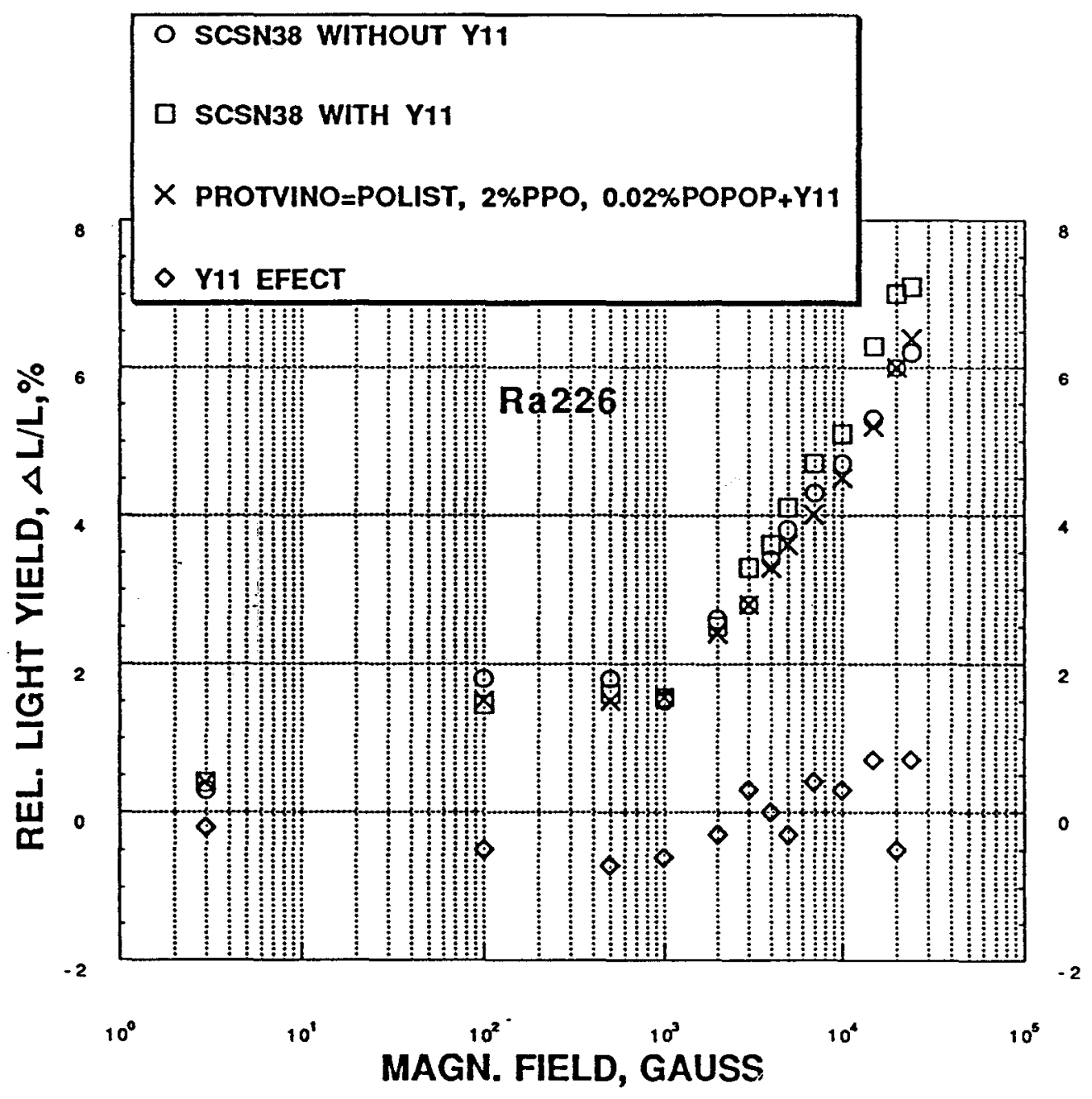

Fig 3. 


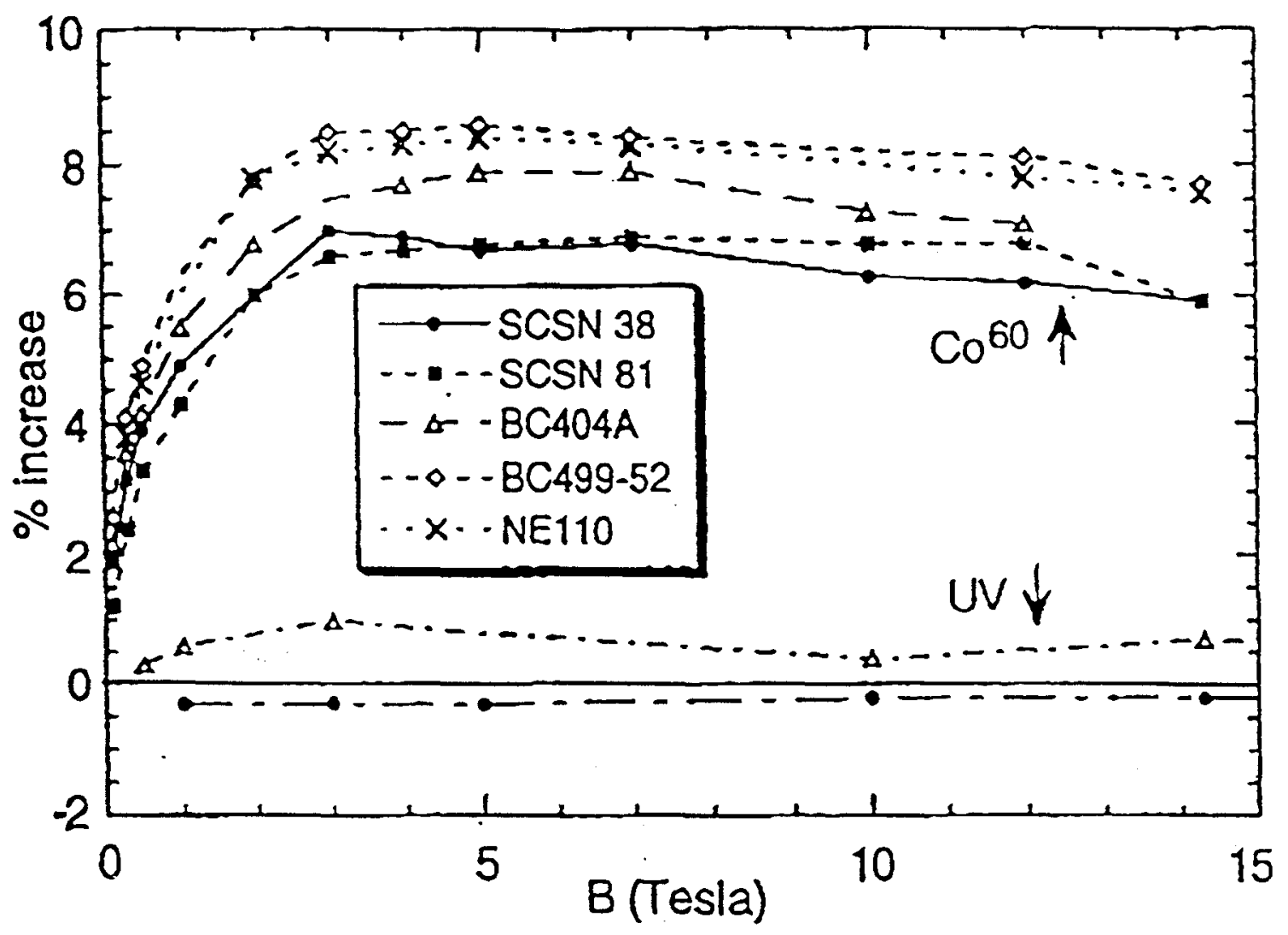

Fig 4 


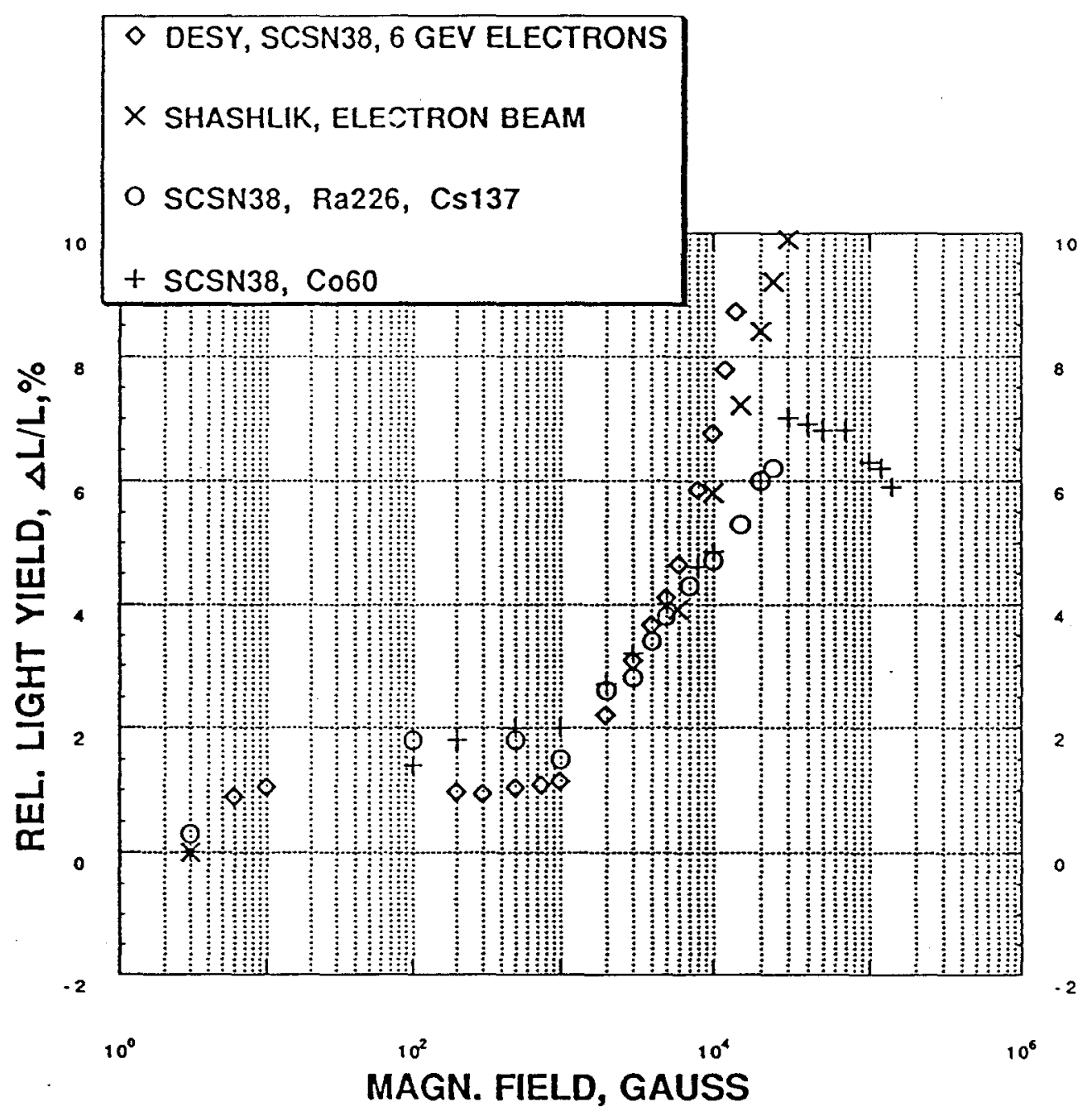

Fiq. 5. 